\title{
Development of Instrument Transmitter Protecting Device against High-Temperature Condition during Severe Accidents
}

\author{
Min Yoo, Sung Min Shin, and Hyun Gook Kang \\ Nuclear Plant Reliability \& Man-Machine Interface Design Laboratory, Korea Advanced Institute of Science and Technology, \\ Daehak-ro 291, Yuseong-Gu, Daejeon 305-701, Republic of Korea
}

Correspondence should be addressed to Hyun Gook Kang; hyungook@kaist.ac.kr

Received 9 March 2014; Accepted 3 May 2014; Published 17 June 2014

Academic Editor: Inn Seock Kim

Copyright (C) 2014 Min Yoo et al. This is an open access article distributed under the Creative Commons Attribution License, which permits unrestricted use, distribution, and reproduction in any medium, provided the original work is properly cited.

Reliable information through instrumentation systems is essential in mitigating severe accidents such as the one that occurred at the Fukushima Daiichi nuclear power plant. There are five elements which might pose a potential threat to the reliability of parameter detection at nuclear power plants during a severe accident: high temperature, high pressure, high humidity, high radiation, and missiles generated during the evolution of a severe accident. Of these, high temperature apparently poses the most serious threat, since thin shielding can get rid of pressure, humidity, radiation (specifically, alpha and beta radiations), and missile effects. In view of this fact, our study focused on designing an instrument transmitter protecting device that can eliminate the high-temperature effect on transmitters to maintain their functional integrity. We present herein a novel concept for designing such a device in terms of heat transfer model that takes into account various heat transfer mechanisms associated with the device.

\section{Introduction}

On March 11, 2011, one of the most serious accidents in nuclear power history took place at the Fukushima Daiichi nuclear power plant as a result of the extreme natural disaster caused by an earthquake and subsequent tsunami [1-3]. The emergency response manuals for severe accidents at the Tokyo Electric Power Company (TEPCO) were developed based on the assumption that the monitoring systems would be normally operating during the severe accidents. However, during the actual accidents at Fukushima they lost detectors and monitoring equipment, so that "the decisions and responses to the accident had to be made on the spot by operational staff at the site, with absent valid tools and manuals" [4]. Without the information on the plant operation, monitoring the process parameters such as temperature, pressure, water level, or radiation was extremely difficult.

There are some detectors that are needed in mitigating severe accidents. For instance, the following are referred to as requisite detectors in the severe accident management guidance (SAMG) for a pressurized water reactor (PWR): core exit thermocouple (CET), heated junction thermocouple (HJTC), resistance temperature detector (RTD), pressurizer manometer, safety injection flow meter, auxiliary feedwater flow meter, steam generator water level gauges, water level gauges for in-containment refueling water storage tank (IRWST), hydrogen sensor, radiation sensor, containment pressure sensor, containment temperature sensor, and containment spray flow meter [5]. Among requisite detectors, thermocouple, RTD, pressure sensor, and radiation sensor are exposed to high temperature. Thermocouple (TC) and RTD sensor do not need to be protected once the temperature is below melting point. TC, RTD, and radiation sensor are expected to lose their accuracy if they are protected by the device. Pressure sensor transmitter includes pressure sensing function. Pressure is directly put to the transmitter from the place where it should be measured through pipe; then transmitter produces electric signal. Thus the study aims to protect the transmitter (pressure sensor and RTD) from high temperature.

A transmitter in an instrumentation system converts analog signals from a sensor to a few $\mathrm{mA}$ electronic signals. Then, those signals can be transferred over long distance with little noise. Among the requisite detectors, manometer, flow meter, and water level gauges have a transmitter. In a severe accident, transmitters may be out of control in 
harsh environment. They are not manufactured so that they can endure in harsh environmental conditions. One of the transmitters that supports pressure detectors endures (a) 1 hour at $157.8^{\circ} \mathrm{C}$ and 4.826 bar; (b) 7 hours at $150.5^{\circ} \mathrm{C}$ and 3.819 bar; and (c) 42 hours at $110^{\circ} \mathrm{C}$ and 0.414 bar steam exposure, with an accuracy within $\pm 0.75 \%$ [6]. In the case of other transmitters, the long-term limitation of temperature over a few tens of hours is $80^{\circ} \mathrm{C}$ with some safety margin.

Based on the severe accident analysis for Shin Kori Units 3 and 4 PWRs, the temperature and pressure around the transmitters during severe accidents are too high to endure. One of the most harsh compartment temperatures reaches $600^{\circ} \mathrm{C}$ right after the accident occurrence and then decreases to $180^{\circ} \mathrm{C}$ during the first 10 minutes remaining around this temperature afterwards. Under this harsh environmental condition associated with high temperature, pressure, humidity, or radiation, the transmitters are not likely to perform their intended functions. In addition, they also may be subject to missiles generated during a severe accident. Each of these five elements poses a potential threat to the reliability of parameter detection at nuclear power plants.

Therefore, in order that instrument transmitters can properly send signals during a severe accident, they must be protected against such harsh environment as might be caused during the evolution of such an accident. Of the aforementioned five elements, this research focuses on protection of transmitters from high temperature. The reason for this focus is that high temperature is the most serious threat, since thin shielding can get rid of pressure, humidity, radiation (alpha and beta), and missile effects.

This research is specially aimed at maintaining the temperature of instrument transmitters below the long-term limitation temperature mentioned above, that is, $80^{\circ} \mathrm{C}$, for at least 72 hours in the harsh environmental conditions. The duration of 72 hours is in line with a typical assumption that if the accident condition is managed for 72 hours, core damage is not likely to occur in a nuclear power plant $[7,8]$.

In the sequel, we present a novel concept to design a protecting device for instrument transmitters in hightemperature environmental condition. The structural design scheme of a cooler is first discussed along with the theoretical heat transfer model. Cooling methods are then described taking into account various factors affecting the protector performance, such as the protector thickness, material, size, environmental pressure, inside heat generation from the transmitter, and environmental temperature.

\section{Design Concept for Instrument Transmitter Protecting Device}

According to a study performed for APR1400 by Korea Hydro and Nuclear Power Company (KHNP), the temperature in some compartments at the APR1400 plant for accident scenarios such as loss of feedwater flow (LOFW), loss of coolant accident (LOCA), or station blackout (SBO) reaches as high as $600^{\circ} \mathrm{C}$ for 10 seconds into the initiating event, drops to around $180^{\circ} \mathrm{C}$ in 600 seconds, and then remains at $180^{\circ} \mathrm{C}$ [5]. Therefore, in order to protect the integrity of transmitters,

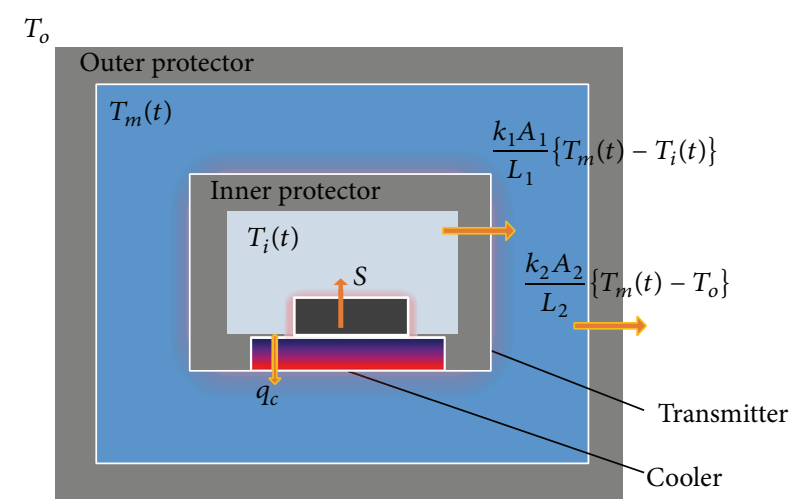

FIGURE 1: Instrument transmitter protecting device.

we envision that the critical transmitters should be protected by a box-shaped protecting device for insulation.

2.1. Cooler and Cooling Method. Figure 1 shows a schematic of the protecting device. The protection system consists of inner and outer protectors in the form of a double-box shape. Transmitters are placed in the interior of the inner protector surrounded by air. The space between the outer protector and the inner protector is filled with water. The outer protector shields heat from the outside, and the inner protector releases heat from inside heat source. The water contained between the two protectors stores heat from both the inside and the outside. A cooler is optionally installed. We derive an equation for the inner temperature from heat transfer relations with an aim to determine appropriate protector properties, sizes, amount of water, and so on.

$T_{i}(t)$ and $T_{m}(t)$ represent the temperature inside the inner protector and the intermediate water temperature, respectively. $S$ is heat generation from the inside (i.e., transmitter), and $(k A / L) \Delta T$ represents the heat transfer by conduction due to temperature difference between the inside and the outside protectors. $q_{c}$ represents heat removal by the cooler. Subscripts 1 and 2 refer to the inner and the outer protector, respectively.

There are a few cooling methods available based on use of heat conduction, refrigerant, vortex tube, and thermoelectric cooler (TEC). However, spatial limitation and harsh environmental condition should be taken into account in designing the instrument transmitter protecting device. Furthermore, the cooling method that will be applied to such devices ought to have high reliability. In consideration of these various constraints, only TEC was judged to be a feasible method in this research. The TEC is based on Peltier effect which is a thermoelectric phenomenon where current flows at junction of two different conductors; one side is heated and the other side is cooled. Figure 2 is a general structure of single stage TEC [9]. It consists of insulators (ceramics plates), soldering, semiconductors (pellets), and electric conductors $[9,10]$. 


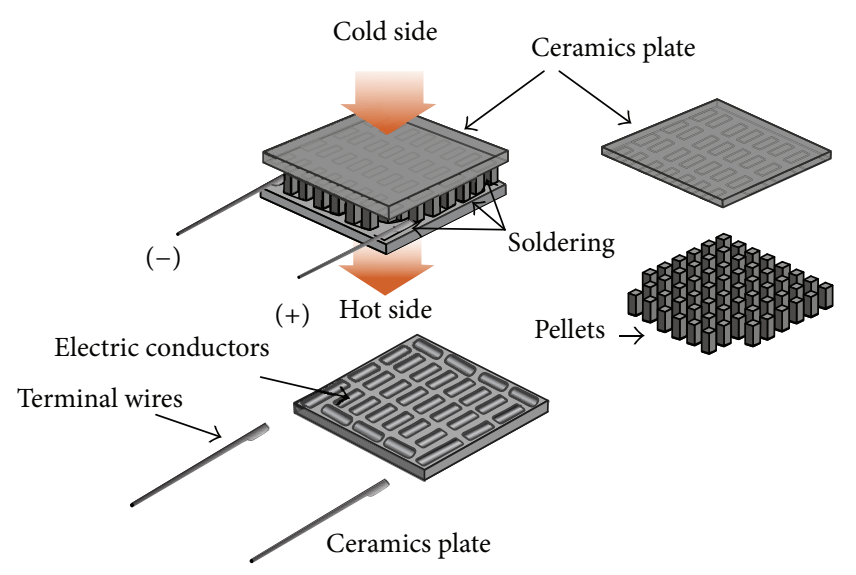

Figure 2: Structure of TEC.

The heat pumped at cold surface of the TEC can be expressed as

$$
q_{c}=2 N\left[\alpha I T_{c}-\left(\frac{I^{2} \rho}{(2 G)}\right)-k_{c}\left(T_{h}-T_{c}\right) G\right],
$$

where $N$ is the number of thermocouples, $\alpha$ is Seebeck coefficient, $T_{c}$ and $T_{h}$ are cold and hot side temperatures, $I$ is current, $G$ is the area divided by the length of the element, and $k_{c}$ is thermal conductivity. $\alpha, G$, and $k_{c}$ are constants related to the TEC material properties [11].

In this study, the inner temperature of the protector system is derived based on the following assumptions.

(1) Convections are ignorable and, as a result, the surface temperature of the protector is assumed to equal the temperature of the fluid that it faces. Only conductive heat transfer works through the wall. That is, the heat transfer rate equals $(k A / L) \Delta T$.

(2) It is reasonable to assume that the ambient temperature $T_{o}$ is constant. The initial thermal shock has a negligible impact on $T_{i}(t)$.

(3) The initial temperature of all materials and medium is $T(0)=20^{\circ} \mathrm{C}$.

(4) The temperature of the protector wall changes linearly along the wall thickness. $T(0, t)$ is the outer surface temperature of the wall at time $t$, and $T(L, t)$ is inner surface temperature of the wall at time $t$. $L$ is thickness of the wall, and with wall temperature along thickness $x$ and time $t, T(x, t)$ equals $((T(L, t)-T(0, t)) / L) x+$ $T(0, t)$.

(5) The outside size of the inner protector is assumed to be $l_{11} \times l_{12} \times l_{13}=0.3 \times 0.3 \times 0.4 \mathrm{~m}^{3}$.

(6) The width, length, and height of outer protector are $l_{21}=l_{11}+l, l_{22}=l_{12}+l$, and $l_{23}=l_{13}+l$, where $l$ is length difference between the inner and outer protector edges.
(7) The temperature of the protector wall is a volumetric average temperature of $T(0, t)$ and $T(L, t)$. It means that average temperature is

$$
\begin{aligned}
& T_{\text {avg }} \begin{array}{l}
\int T(x, t) d V \\
=\frac{\text { wall volume } V}{L} \\
=\left(\int_{0}^{L}\left\{\frac{T(L, t)-T(0, t)}{L} x+T(0, t)\right\}\right. \\
\quad \times 2\left\{\left(l_{1}-2 x\right)\left(l_{2}-2 x\right)\right. \\
\quad+\left(l_{2}-2 x\right)\left(l_{3}-2 x\right) \\
\left.\left.\quad+\left(l_{3}-2 x\right)\left(l_{1}-2 x\right)\right\} d x\right) \\
\quad \times\left(l_{1} l_{2} l_{3}-\left(l_{1}-2 L\right)\left(l_{2}-2 L\right)\left(l_{3}-2 L\right)\right)^{-1} \\
=\left(T(L, t)\left\{3 L^{2}-\frac{4}{3}\left(l_{1}+l_{2}+l_{3}\right) L+\frac{1}{2}\left(l_{1} l_{2}+l_{2} l_{3}+l_{3} l_{1}\right)\right\}\right. \\
\left.\quad+T(0, t)\left\{L^{2}-\frac{2}{3}\left(l_{1}+l_{2}+l_{3}\right) L+\frac{1}{2}\left(l_{1} l_{2}+l_{2} l_{3}+l_{3} l_{1}\right)\right\}\right) \\
\quad \times\left(4 L^{2}-2\left(l_{1}+l_{2}+l_{3}\right) L+\left(l_{1} l_{2}+l_{2} l_{3}+l_{3} l_{1}\right)\right)^{-1} \\
=\alpha T(0, t)+\beta T(L, t),
\end{array}
\end{aligned}
$$

where

$$
\begin{gathered}
\alpha=\frac{L^{2}-(2 / 3)\left(l_{1}+l_{2}+l_{3}\right) L+(1 / 2)\left(l_{1} l_{2}+l_{2} l_{3}+l_{3} l_{1}\right)}{4 L^{2}-2\left(l_{1}+l_{2}+l_{3}\right) L+\left(l_{1} l_{2}+l_{2} l_{3}+l_{3} l_{1}\right)}, \\
\beta=\frac{3 L^{2}-(4 / 3)\left(l_{1}+l_{2}+l_{3}\right) L+(1 / 2)\left(l_{1} l_{2}+l_{2} l_{3}+l_{3} l_{1}\right)}{4 L^{2}-2\left(l_{1}+l_{2}+l_{3}\right) L+\left(l_{1} l_{2}+l_{2} l_{3}+l_{3} l_{1}\right)} .
\end{gathered}
$$

$\alpha$ and $\beta$ are about 0.5 unless wall thickness is too thick.

2.2. Heat Transfer Model. Whether the instrument transmitter protection system successfully carries out its intended function or not depends on the inner temperature. That is, the maximum $T_{i}(t)$ should remain below $80^{\circ} \mathrm{C}$ for the period of 72 hours.

Equation (1) can be revised to (5) under the aforementioned assumptions and $e_{1}=2 N\left(\alpha I+k_{c} G\right), e_{2}=2 N k_{c} G$, and $e_{3}=\rho I^{2} N / G$.

Consider

$$
q_{c}=e_{1} T_{i}(t)-e_{2} T_{m}(t)-e_{3} .
$$


The first part of the derivation of inside the inner protector is about temperature change in the intermediate water:

$$
\begin{aligned}
\frac{k_{1} A_{1}}{L_{1}} & \left\{T_{i}(t)-T_{m}(t)\right\}+\frac{k_{2} A_{2}}{L_{2}}\left\{T_{o}-T_{m}(t)\right\}+q_{c} \\
& =c_{m} m_{m} \dot{T}_{m}+c_{2} m_{2} \frac{d}{d x}\left(\frac{\int_{0}^{L_{2}} T_{2, \text { avg }}(x, t) d V}{V_{2}}\right) \\
& =\left(c_{m} m_{m}+c_{2} m_{2} \beta_{2}\right) \dot{T}_{m},
\end{aligned}
$$

where $k$ is thermal conductivity, $A$ the surface area, and $L$ the thickness of the protector. $c_{m}$ and $m_{m}$ are the specific heat capacity and mass of water, respectively, and $\dot{T}_{m}$ is time derivative of water temperature. $\beta_{2}$ equals $\left(3 L_{2}^{2}-(4 / 3)\left(l_{21}+\right.\right.$ $\left.\left.l_{22}+l_{23}\right) L_{2}+(1 / 2)\left(l_{21} l_{22}+l_{22} l_{23}+l_{23} l_{21}\right)\right) /\left(4 L_{2}^{2}-2\left(l_{21}+l_{22}+\right.\right.$ $\left.\left.l_{23}\right) L_{2}+\left(l_{21} l_{22}+l_{22} l_{23}+l_{23} l_{21}\right)\right)$.

$$
\begin{aligned}
& \text { If } \\
& \begin{aligned}
a_{1} & =\frac{k_{1} A_{1}}{L_{1}}, \quad a_{2}=\frac{k_{2} A_{2}}{L_{2}}, \\
b_{1} & =\frac{a_{1}+e_{1}}{c_{m} m_{m}+c_{2} m_{2} \beta_{2}}, \quad b_{2}=\frac{a_{2} T_{o}-e_{3}}{c_{m} m_{m}+c_{2} m_{2} \beta_{2}}, \\
p & =\frac{a_{1}+a_{2}+e_{2}}{c_{m} m_{m}+c_{2} m_{2} \beta_{2}},
\end{aligned}
\end{aligned}
$$

from (6), the intermediate water temperature becomes

$$
T_{m}(t)=e^{-p t}\left[T(0)+\int_{0}^{t} e^{p \tau}\left\{b_{1} T_{i}(\tau)+b_{2}\right\} d \tau\right] .
$$

The second part of the derivation is about a temperature change in the inner protector and the inside temperature change, $c_{i} m_{i} \ll c_{1} m_{1}$ :

$$
\begin{aligned}
S+ & a_{1}\left\{T_{m}(t)-T_{i}(t)\right\}-q_{c} \\
& =c_{i} m_{i} \dot{T}_{i}+c_{1} m_{1} \frac{d}{d x}\left(\frac{\int_{0}^{L_{1}} T_{1, \mathrm{avg}}(x, t) d V}{V_{1}}\right) \\
& \sim c_{1} m_{1}\left(\alpha_{1} \dot{T}_{m}+\beta_{1} \dot{T}_{i}\right),
\end{aligned}
$$

where

$$
\begin{aligned}
& \alpha \\
& =\frac{L_{1}^{2}-(2 / 3)\left(l_{11}+l_{12}+l_{13}\right) L_{1}+(1 / 2)\left(l_{11} l_{12}+l_{12} l_{13}+l_{13} l_{11}\right)}{4 L_{1}^{2}-2\left(l_{11}+l_{12}+l_{13}\right) L_{1}+\left(l_{11} l_{12}+l_{12} l_{13}+l_{13} l_{11}\right)}, \\
& \beta_{1} \\
& =\frac{3 L_{1}^{2}-(4 / 3)\left(l_{11}+l_{12}+l_{13}\right) L_{1}+(1 / 2)\left(l_{11} l_{12}+l_{12} l_{13}+l_{13} l_{11}\right)}{4 L_{1}^{2}-2\left(l_{11}+l_{12}+l_{13}\right) L_{1}+\left(l_{11} l_{12}+l_{12} l_{13}+l_{13} l_{11}\right)} .
\end{aligned}
$$

Substituting (5) and (8) into (9) results in

$$
A \ddot{T}_{i}+B \dot{T}_{i}+C T_{i}=p\left(S+e_{3}\right)+b_{2}\left(a_{1}+e_{2}\right) .
$$

This is a second-order nonhomogeneous differential equation, where

$$
\begin{aligned}
A= & c_{i} m_{i}+c_{1} m_{1} \beta_{1} \sim c_{1} m_{1} \beta_{1}, \\
B= & p A+\alpha_{1} b_{1} c_{1} m_{1}+a_{1} \\
& +e_{1} \sim \frac{a_{1}+a_{2}+e_{2}}{c_{m} m_{m}+c_{2} m_{2} \beta_{2}} * c_{1} m_{1} \beta_{1} \\
& +\frac{\alpha_{1}\left(a_{1}+e_{1}\right) c_{1} m_{1}}{c_{m} m_{m}+c_{2} m_{2} \beta_{2}}+a_{1}+e_{1}, \\
C= & a_{2} b_{1} .
\end{aligned}
$$

Because $B^{2}-4 A C$ is always positive, its general solution is

$$
T_{i}(t)=d_{1} e^{r_{1} t}+d_{2} e^{r_{2} t}+d_{3}
$$

$d_{1}$ and $d_{2}$ are arbitrary constants and $r_{1}$ and $r_{2}$ are $(-B \mp$ $\left.\sqrt{B^{2}-4 A C}\right) / 2 A$; they are always negative. $d_{3}$ equals $\left(1 /\left(a_{1}+\right.\right.$ $\left.\left.e_{1}\right)\right)\left\{\left(a_{1}+e_{2}\right) T_{o}+e_{3}\right\}+\left(a_{1}+a_{2}+e_{2}\right) /\left(a_{2}\left(a_{1}+e_{1}\right)\right) S$. The first term can be eliminated as $r_{1} \ll r_{2}$ and $d_{2}$ equals $T(0)-d_{3}$ because $T_{i}(t)=T(0)$. Equation (13) becomes

$$
\begin{aligned}
& T_{i}(t) \\
& =\left\{T(0)-d_{3}\right\} e^{r_{2} t}+d_{3} \\
& =\left[T(0)-\left(\frac{1}{a_{1}+e_{1}}\left\{\left(a_{1}+e_{2}\right) T_{o}+e_{3}\right\}+\frac{a_{1}+a_{2}+e_{2}}{a_{2}\left(a_{1}+e_{1}\right)}\right)\right] \\
& \quad \times e^{\left(\left(-B+\sqrt{B^{2}-4 A C}\right) / 2 A\right) t} \\
& \quad+\frac{1}{a_{1}+e_{1}}\left\{\left(a_{1}+e_{2}\right) T_{o}+e_{3}\right\}+\frac{a_{1}+a_{2}+e_{2}}{a_{2}\left(a_{1}+e_{1}\right)} S .
\end{aligned}
$$

\section{Results and Discussion}

The temperature inside the inner protector, that is, $T_{i}(t)$, continues to increase and converges at $d_{3}$. Thus, its maximum temperature, that is, $T_{i}(72 \mathrm{hr})$, must be smaller than the limiting temperature $T_{\lim }$ of $80^{\circ} \mathrm{C}$, as discussed above:

$$
T_{i}(72 \mathrm{hr}) \leq T_{\text {lim }}
$$

The variables affecting this criterion are the outer protector size/thickness/thermal conductivity, the inner protector thickness/thermal conductivity/heat capacity, and the number of cooler/current supplies. There are too many variables to derive the appropriate protector condition. Thus, some variables such as material properties were fixed in this analysis for the sake of computational simplification. Further analyses will be performed with different assumptions as deemed necessary in the future study.

There arises an issue whether the protection system needs to include a cooler. Regardless of whether to include a cooler or not, it will be good to use small $a_{2}$ and large size of outer protector. If the inner protector is strongly insulated, the system will need a cooler to remove heat from the inside of 
the inner protector to the water. In this case, the smaller $a_{1}$, the larger the number of TECs, and the higher the current, the better. Equation (16) is a simplified form of (14) applying $c_{m} m_{m}+c_{2} m_{2} \beta_{2} \gg c_{1} m_{1}$. Consider

$$
\begin{aligned}
& T_{i}(t) \\
& \sim\left[T(0)-\left(\frac{1}{a_{1}+e_{1}}\left\{\left(a_{1}+e_{2}\right) T_{o}+e_{3}\right\}+\frac{a_{1}+a_{2}+e_{2}}{a_{2}\left(a_{1}+e_{1}\right)} S\right)\right] \\
& \quad \times e^{\left(\left(-\left(a_{1}+e_{1}\right)+\sqrt{\left(a_{1}+e_{1}\right)^{2}-2 a_{1} a_{2}\left(c_{1} m_{1} /\left(c_{m} m_{m}+c_{2} m_{2} / 2\right)\right)}\right) / c_{1} m_{1}\right) t} \\
& \quad+\frac{1}{a_{1}+e_{1}}\left\{\left(a_{1}+e_{2}\right) T_{o}+e_{3}\right\}+\frac{a_{1}+a_{2}+e_{2}}{a_{2}\left(a_{1}+e_{1}\right)} S .
\end{aligned}
$$

In the case where no cooler is used, heat should be well transferred between the inner air and the water because the heat from the transmitter itself is accumulated inside the air. The outer protector needs a strong insulation in both cases to protect heat invasion from the environment. Hence, $a_{1} \gg a_{2}$, $c_{m} m_{m}+c_{2} m_{2} \beta_{2} \gg c_{1} m_{1}$, and $e_{1}=e_{2}=e_{3}=0$. It is reasonable to assume that $\beta_{1}$ and $\beta_{2}$ are 0.5 each. Equation (14) becomes

$$
\begin{aligned}
& T_{i}(t) \\
& \sim\left[T(0)-\left\{T_{o}+\frac{S}{a_{2}}\right\}\right] \\
& \quad \times e^{\left(\left(-a_{1}+\sqrt{\left(a_{1}\right)^{2}-2 a_{1} a_{2}\left(c_{1} m_{1} /\left(c_{m} m_{m}+c_{2} m_{2} / 2\right)\right)}\right) / c_{1} m_{1}\right) t} \\
& \quad+T_{o}+\frac{S}{a_{2}} .
\end{aligned}
$$

The inner protector wall material and thickness are much less influential factors than other variables as long as inner protector wall material has high thermal conductivity. A material that has $1 \mathrm{~J} / \mathrm{g} / \mathrm{K}$ specific heat, $320 \mathrm{~g} / \mathrm{m}^{3}$ density, $5 \mathrm{~W} / \mathrm{m} / \mathrm{K}$ thermal conductivity, and $1 \mathrm{~cm}$ thickness is applied to the inner protector wall. Outer protector material is one of the best insulations whose specific heat, density, and thermal conductivity are $0.8 \mathrm{~J} / \mathrm{g} / \mathrm{K}, 250 \mathrm{~kg} / \mathrm{m}^{3}$, and $0.25 \mathrm{~W} / \mathrm{m} / \mathrm{K}$, respectively $[12,13]$.

Figure 3 is a top view of 3 -dimensional plot of $T_{i}(t)$ when $t$ is 72 hours in (17). The $y$-axis in this figure is the length difference between the outer protector and the inner protector. The $x$-axis represents the thickness of the outer protector wall. The area above the line indicates that $T_{i}(t)$ is below $80^{\circ} \mathrm{C}$ and the area below the line indicates that the temperature is higher than $80^{\circ} \mathrm{C}$. The protector has the minimum size at the minimum point $(0.066,0.278)$ of the line. Then, the outer protector size becomes $0.578 \times 0.578 \times$ $0.678 \mathrm{~m}^{3}$ with $6.6 \mathrm{~cm}$ thickness. The lower and right part calculations of Figure 3 are meaningless because the outer wall and the inner wall are overlapped.

Figure 4 is the temperature distribution at 72 hours after the accident simulated by solidworks flow simulation. Figure 5 shows the inner air temperature along time, comparing equation-based and simulation-based calculations.

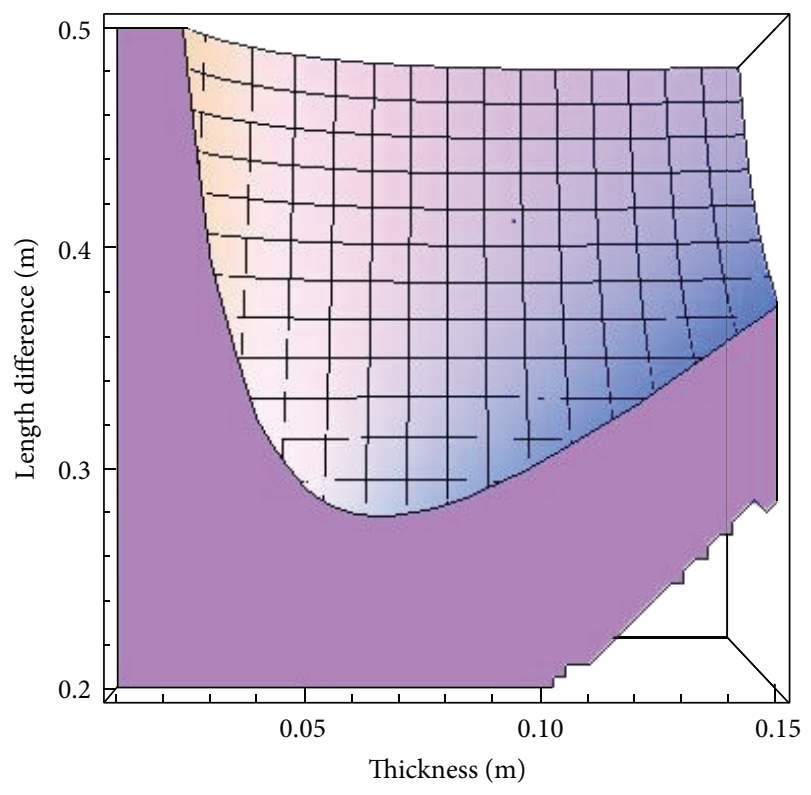

FIGURE 3: $T_{i}(t)$ along wall thickness and length difference between the inner and outer protectors.

The equation-based result yields a conservative result. The temperature reaches $70.99^{\circ} \mathrm{C}$ at 72 hours in the case of the simulation. The temperature profile difference between the equation-based and simulation-based calculations mainly comes from assumptions (1) and (3) described above. The temperature based on heat transfer equations increases more rapidly than the one based on the simulation during the initial phase, because the equation does not consider transient heat transfer and the heat capacity of the wall is underestimated. In assumption (1), it was presumed that there is no convective heat transfer and so the heat transfer between the solid surface and the fluid was assumed to be perfect. However, there is a heat transfer lag in real world and the simulation took into account this phenomenon. Figure 5 overall indicates that the increasing rate of the inner air temperature as predicted by the heat transfer equations quite well corresponds to the one as evaluated by the simulation, although there is a slight difference.

\section{Conclusion}

Reliable information through instrumentation systems is essential in mitigating severe accidents such as the one that occurred at the Fukushima Daiichi nuclear power plant. Thermal-hydraulic analyses performed for several major accident scenarios at a PWR plant, including LOFW, LOCA, and $\mathrm{SBO}$, indicate that compartment temperature reaches $600^{\circ} \mathrm{C}$ in the worst case, although it decreases to $180^{\circ} \mathrm{C}$ in about 10 minutes. The instrument transmitters cannot perform their intended functions under such high-temperature condition.

In addition to high temperature, the instrumentation systems may also be required to function in harsh condition involving high pressure, high humidity, high radiation, and 

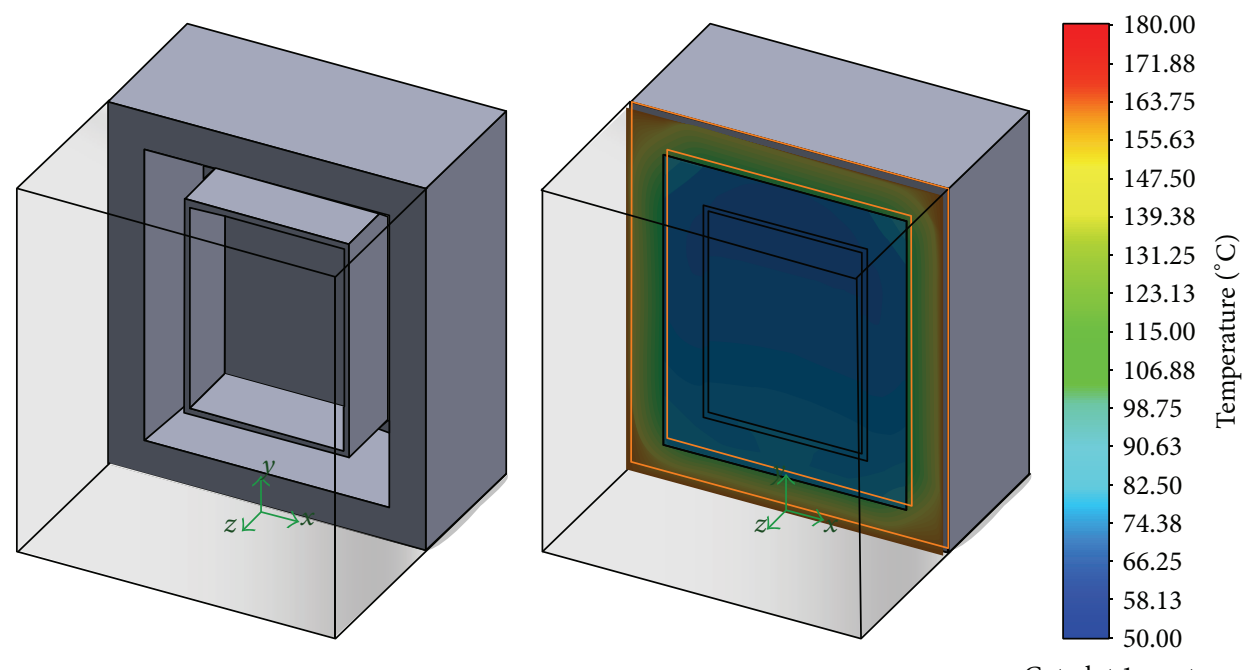

FIGURE 4: The result of simulation and temperature distribution at $t=72$ hours.

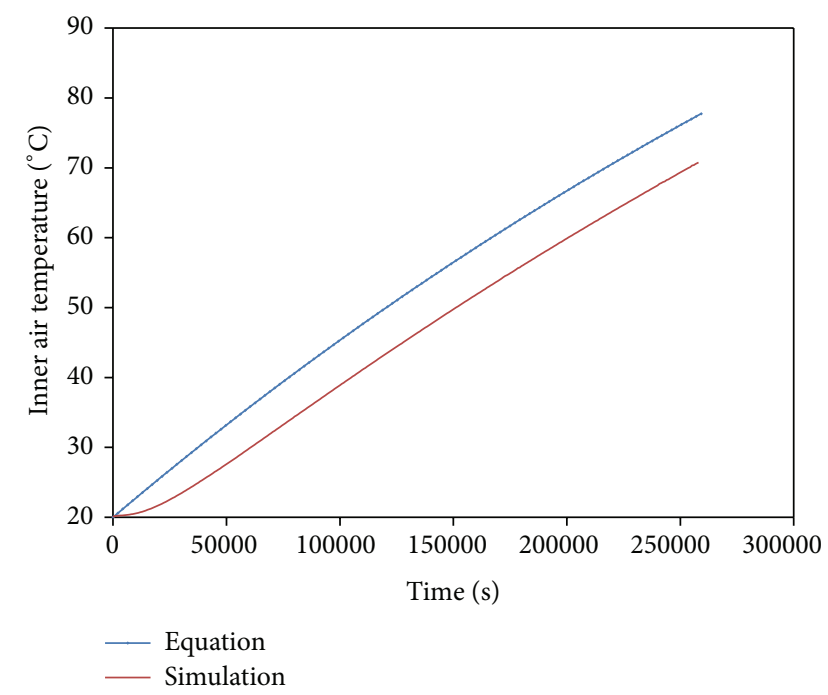

FIgURE 5: Temperature comparison of the results between equationbased and simulation-based calculations along time.

missiles generated during a severe accident. All those five elements pose a potential threat to the reliability of parameter detection at nuclear power plants. In this study, an analysis was carried out to design an instrument transmitter protecting device that can withstand harsh environment, especially high-temperature condition. Of the various threats to the instrumentation system, high temperature apparently poses the most serious threat, since thin shielding can get rid of pressure, humidity, radiation (alpha and beta), and missile effects.

In this study, a novel concept for designing an instrument transmitter protecting device was developed and investigated by analyzing the heat transfer mechanisms. The protection system may be developed either with or without a cooler, and the design without a cooler turns out to be more effective.
The thermal properties and geometry of the protector material also influence the result. Thermal conductivity controls heat conduction itself; on the other hand, heat capacity of the material controls heat spreading by saving heat in the material. The inside heat generation has impact on long-term temperature and heat accumulation. So less heat generating equipment had better be considered. Our study also points out that transient heat transfer and convective heat transfer should be considered to avoid excessively conservatism in the analysis and as a result, obtain a more accurate solution.

Lastly, note that although transmitters can be easily protected from alpha and beta radiations due to the water included in the transmitter protecting device, gamma radiation effects ought to be investigated. The gamma ray dose rate in a reactor was estimated to be larger than $150 \mathrm{~Gy} / \mathrm{h}[14]$. Verification experiment is necessary to investigate protector performance in the gamma radiation environment. The next things to do are finding optimized protector structure and material property by developing a more thorough heat transfer model and verifying it through simulation and experiment.

\section{Nomenclature}

$A_{1}$ : Inner protector area $\left(\mathrm{m}^{2}\right)$

$A_{2}$ : Outer protector area $\left(\mathrm{m}^{2}\right)$

$c_{1}$ : Specific heat of inner protector $(\mathrm{J} / \mathrm{kg} \cdot \mathrm{K})$

$c_{2}$ : Specific heat of outer protector $(\mathrm{J} / \mathrm{kg} \cdot \mathrm{K})$

$c_{m}$ : Specific heat of intermediate water $(\mathrm{J} / \mathrm{kg} \cdot \mathrm{K})$

$G$ : Geometry factor

I: $\quad$ Current (amps)

$k_{1}$ : Inner protector thermal conductivity

$(\mathrm{W} / \mathrm{m} \cdot \mathrm{K})$

$k_{2}$ : Outer protector thermal conductivity $(\mathrm{W} / \mathrm{m} \cdot \mathrm{K})$

$k_{c}$ : Thermal conductivity of TEC $(\mathrm{W} / \mathrm{m} \cdot \mathrm{K})$ 
$l$ : $\quad$ Length difference between inner and outer protector edges $(\mathrm{m})$

$l_{11}, l_{12}, l_{13}$ : Inner protector length, width, and height $(\mathrm{m})$

$l_{21}, l_{22}, l_{23}$ : Outer protector length, width, and height

(m)

$L_{1}: \quad$ Inner protector thickness (m)

$L_{2}$ : $\quad$ Outer protector thickness (m)

$m_{1}$ : $\quad$ Mass of inner protector $(\mathrm{kg})$

$m_{2}$ : $\quad$ Mass of outer protector $(\mathrm{kg})$

$m_{m}: \quad$ Mass of intermediate water $(\mathrm{kg})$

$N: \quad$ Number of TECs

$T_{c}: \quad$ Cold side temperature $\left({ }^{\circ} \mathrm{C}\right)$

$T_{h}$ : $\quad$ Hot side temperature $\left({ }^{\circ} \mathrm{C}\right)$

$T_{i}$ : $\quad$ Inside air temperature $\left({ }^{\circ} \mathrm{C}\right)$

$T_{\text {lim }}$ : $\quad$ Limit temperature $\left({ }^{\circ} \mathrm{C}\right)$

$T_{m}: \quad$ Intermediate water temperature $\left({ }^{\circ} \mathrm{C}\right)$

$T_{o}: \quad$ Ambient temperature $\left({ }^{\circ} \mathrm{C}\right)$

$T(0): \quad$ Initial temperature $\left({ }^{\circ} \mathrm{C}\right)$

$S: \quad$ Heat source $(\mathrm{W})$.

\section{Greek Letters}

$\alpha$ : Seebeck coefficient $(\mathrm{V} / \mathrm{K})$

$\rho$ : Resistivity $(\Omega \cdot \mathrm{m})$.

\section{Conflict of Interests}

The authors declare that there is no conflict of interests regarding the publication of this paper.

\section{Acknowledgments}

This work was supported by the International Cooperation of the Korea Institute of Energy Technology Evaluation and Planning (KETEP) under Grant funded by the Korea government Ministry of Trade, Industry and Energy (no. 20121610100030).

\section{References}

[1] OECD/NEA, The Fukushima Daiichi Nuclear Power Plant Accident: OECD/NEA Nuclear Safety Response and Lessons Learnt, Nuclear Energy Agency, Organization for Economic Co-operation and Development, 2013.

[2] C. Miller, A. Cubbage, D. Dorman, J. Grobe, G. Holahan, and N. Sanfilippo, Recommendations for Enhancing Reactor Safety in the 21st Century: The Near-Term Task Force Review of Insights from the Fukushima Dai-Ichi Accident, Nuclear Regulatory Commission, 2011.

[3] Nuclear Emergency Response Headquarters, Report of Japanese Government to the IAEA Ministerial Conference on Nuclear Safety-The Accident at TEPCO's Fukushima Nuclear Power Stations, Nuclear Emergency Response Headquarters, Government of Japan, 2011.

[4] The National Diet of Japan, Fukushima Nuclear Accident Independent Investigation Commission, The Official Report of the Fukushima Nuclear Accident Independent Investigation Commission, The Fukushima Nuclear Accident Independent Investigation Commission, Japan, 2012.
[5] KEPCO and KHNP, APR1400 Design Control Document Tier 2 Chapter 19: Probabilistic Risk Assessment and Severe Accident Evaluation, Korea Electric Power Corporation and Korea Hydro \& Nuclear Power Company, 2013.

[6] Emerson Process Management Rosemount Nuclear Instruments Inc., "Rosemount 1152 alphaline datasheet-nuclear pressure transmitter," Reference Manual, Emerson Process Management Rosemount Nuclear Instruments Inc., Chanhassen, Minn, USA, 2007.

[7] US NRC, Final Safety Evaluation Report Related to Certification of the AP1000 Standard Design, Division of Regulatory Improvement Programs, Office of Nuclear Reactor Regulation, U.S. Nuclear Regulatory Commission, Washington, DC, USA, 2004.

[8] IAEA, "Design measures for prevention and mitigation of severe accidents at advanced water cooled reactors," in Proceedings of the Technical Committee Meeting, Vienna, Austria, October 1996.

[9] TEC Microsystems GmbH, Thermoelectric Cooler Basics, TEC Microsystems GmbH, Berlin, Germany, 2013.

[10] F. J. Disalvo, “Thermoelectric cooling and power generation," Science, vol. 285, no. 5428, pp. 703-706, 1999.

[11] Laird Technologies, Thermoelectric Handbook, Laird Technologies, St. Louis, Mo, USA, 2010.

[12] Microtherm, “Microtherm standard panel," Datasheet, 2012.

[13] Z. Luo, "A simple method to estimate the physical characteristics of a thermoelectric cooler from vendor datasheets," Electronics Cooling, vol. 14, no. 3, pp. 22-27, 2008.

[14] J. W. Cho and K. M. Jeong, "A performance evaluation of a notebook PC under a high dose-rate gamma ray irradiation test," Science and Technology of Nuclear Installations, vol. 2014, Article ID 362354, 8 pages, 2014. 


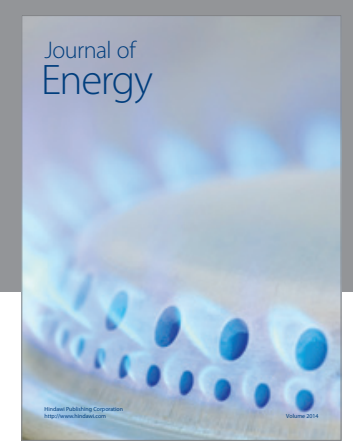

Journal of

Industrial Engineering
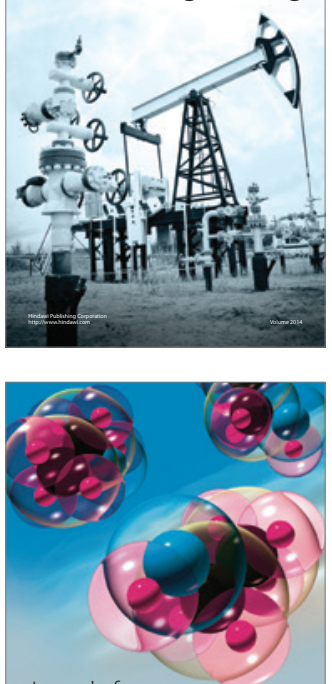

Fuels
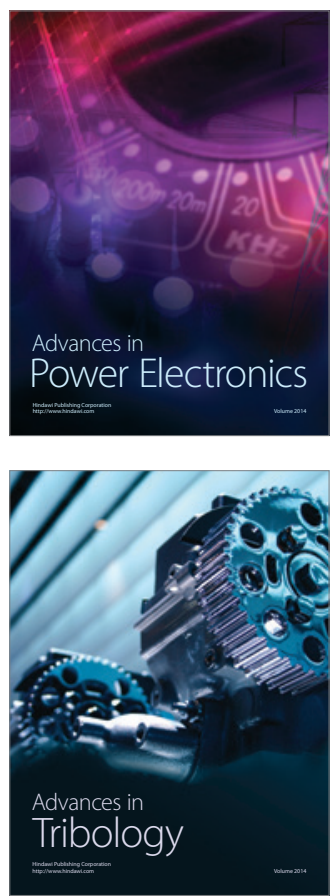

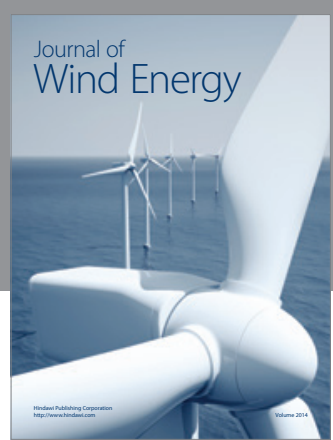

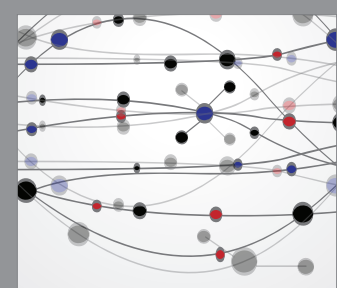

The Scientific World Journal

Submit your manuscripts at http://www.hindawi.com

Journal of

Structures
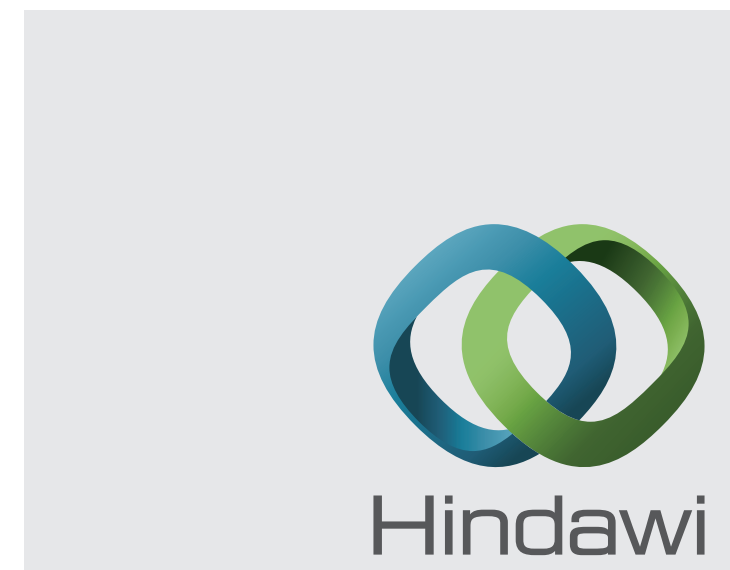

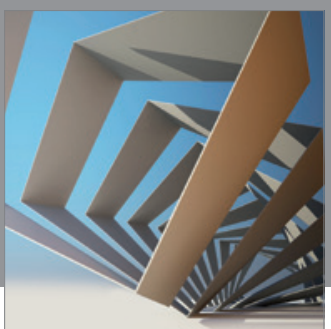

Rotating

Machinery
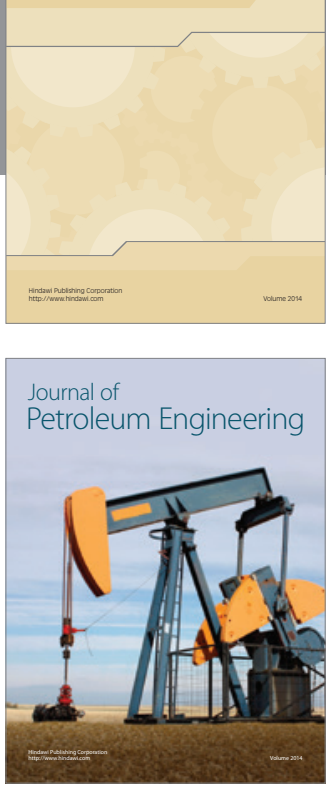

Journal of

Solar Energy
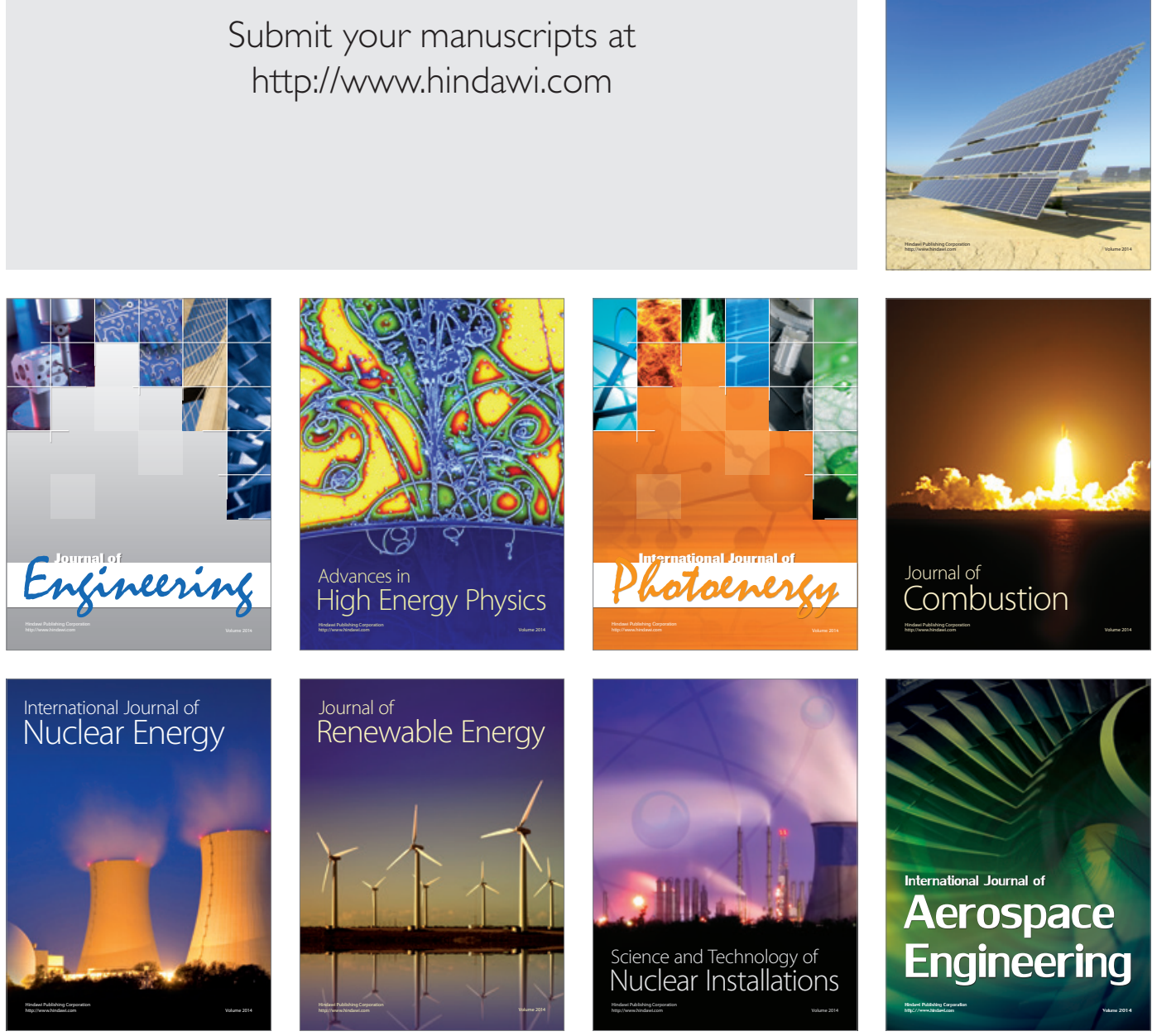